\title{
EFICIÊNCIA DA ALOCAÇÃO DE RECURSOS PÚBLICOS NAS UNIDADES DA FEDERAÇÃO NOS GOVERNOS LULA E DILMA
}

\section{EFFICIENCY OF FEDERAL-TO-STATE ALLOCATION OF RESOURCES DURING ADMINISTRATIONS OF BRAZILIAN PRESIDENTS LULA AND DILMA}

\section{EFICIENCIA EN LA DISTRIBUCIÓN DE RECURSOS PÚBLICOS EN LAS UNIDADES DE LA FEDERACIÓN DURANTE LOS GOBIERNOS LULA Y DILMA}

\begin{abstract}
RESUMO
A eficiência nos gastos constitui um dos principais desafios da gestão pública. Neste artigo, analisa-se a eficiência na alocação de recursos públicos nas 27 Unidades da Federação (UF) durante os governos Lula e Dilma. Para tanto, utilizou-se a Análise Envoltória de Dados (Data Envelopment Analysis - DEA), no modelo Retornos Constantes à Escala, proposto por Charnes, Cooper e Rhodes (1978) e conhecido como modelo CCR, e no modelo Retornos Variáveis à Escala, proposto por Banker, Charnes e Cooper (1984) e nomeado BCC, com orientação de output, para o cálculo de eficiência das 27 UFs. Como entrada para os dois modelos, foram utilizados os gastos com saúde e educação de cada UF, assim como a renda média familiar. Como saída, utilizou-se a média do Índice FIRJAN de Desenvolvimento Municipal (IFDM) dos municípios de cada UF. Foram feitas correlações entre a eficiência de cada UF obtida pela DEA e o respectivo Produto Interno Bruto (PIB). Com base no CCR, em que a eficiência é calculada considerando-se 0 axioma da proporcionalidade entre inputs e outputs, houve maior número de UFs eficientes no governo Dilma. Em relação ao BCC, o qual admite que a eficiência máxima varie em função da economia de escala, os resultados são semelhantes entre os dois governos, apresentando 0 governo Lula maior número de UFs eficientes. Há correlação entre os modelos CCR e BCC, assim como entre o modelo CCR e o PIB, mas não há correlação entre o modelo BCC e o PIB.
\end{abstract}

PALAVRAS-CHAVE: Eficiência, gastos públicos, gestão pública, governo Lula, governo Dilma.

Clayton Robson Moreira da Silva ${ }^{1}$

claytonrmsilva@gmail.com

ORCID: 0000-0003-0717-2713

Roberta Michele Ponte Alves ${ }^{2}$

pontealves@yahoo.com.br

ORCID: 0000-0001-7405-5010

Márcia Martins Mendes De Luca ${ }^{3}$

marciadeluca@ufc.br

ORCID: 0000-0002-9995-5637

Alessandra Carvalho de Vasconcelos ${ }^{3}$

alevasconcelos.ufc@gmail.com

ORCID: 0000-0002-6480-5620

1 Instituto Federal de Educação, Ciência e Tecnologia do Piauí, Campus Pedro II, Eixo de Gestão e Negócios, Pedro II, PI, Brasil

2 Universidade Federal do Ceará, Programa de Pós-Graduação em Administração e Controladoria, Fortaleza, CE, Brasil

3 Universidade Federal do Ceará, Faculdade de Economia, Administração, Atuária e Contabilidade, Departamento de Contabilidade

Submetido 31.01.2018. Aprovado 26.06.2019

Avaliado pelo processo de double blind review

DOI: http://dx.doi.org/10.12660/cgpc.v24n78.73696 
Clayton Robson Moreira da Silva - Roberta Michele Ponte Alves - Márcia Martins Mendes De Luca - Alessandra Carvalho de Vasconcelos

\section{ABSTRACT}

Efficiency in public spending is a major challenge of public administration. In this study, the efficiency of allocation of federal resources to Brazil's 27 states (UFS) during the administrations of presidents Lula and Dilma was assessed. To do so, data envelopment analysis (DEA) to the constant returns to scale model (Charnes, Cooper and Rhodes, 1978), known as CCR model, and the variable returns to scale model (Banker, Charnes and Cooper, 1984), named BCC model, output-oriented were applied. Spending on health care and education and mean household income was used as variables in the models, while the mean FIRJAN index of municipal development (IFDM) of each UF was adopted as outcome parameter. Subsequently, DEA-measured allocation efficiency was correlated with the GDP of each UF. Using CCR model (according to which efficiency is calculated assuming a proportionality between inputs and outputs), the number of efficient UFs was found to be significantly greater during Dilma administration. Using BCC model (according to which maximum efficiency is dependent on economy of scale), the number of efficient UFs was greater during Lula administration, but the difference was non-significant. Correlations were observed between CCR model and BCC model and between CCR model and GDP, but not between BCC model and GDP.

KEYWORDS: Efficiency, public spending, public administration, Lula administration, Dilma administration.

\section{RESUMEN}

La eficiencia en gastos constituye uno de los principales desafíos de la gestión pública. En este artículo, analizamos la eficiencia en la distribucion de recursos públicos en las 27 Unidades de la Federación (UF) durante los gobiernos Lula y Dilma. Para esto, se utilizó el Análisis Envolvente de Datos (Data Envelopment Analysis - DEA), en el modelo Rendimientos Constantes a Escala, propuesto por Charnes, Cooper y Rhodes (1978), denominado modelo CCR; y en el modelo Retornos Variables a Escala, propuesto por Banker, Charnes y Cooper (1984), llamado de BCC, orientado a output, para calcular la eficiencia de las 27 UFs. Como entrada para los dos modelos, se utilizaron los gastos en salud y educación de cada UF, y los ingresos familiares promedio. Como salida, se utilizó el promedio del Indice FIRJAN de Desarrollo Municipal (IFDM) de los municipios de cada UF. Se hicieron correlaciones entre la eficiencia de cada estado, obtenida a través del DEA y el respectivo Producto Interno Bruto (PIB). Basados en el CCR, donde se calcula la eficiencia a partir de la proporcionalidad entre inputs y outputs, hubo más UFs eficientes en el gobierno Dilma. Con relación a BCC, que admite que la eficiencia máxima varíe en función de la economía de escala, los resultados son semejantes entre los dos gobiernos, aunque en el gobierno Lula hubo más UFs eficientes. Hay correlación entre los modelos CCR y BCC, así como entre el modelo CCR y el PIB, pero no hay correlación entre el modelo $B C C$ y el PIB.

PALABRAS CLAVE: Eficiencia, gastos públicos, gestión pública, gobierno Lula, gobierno Dilma.

\section{INTRODUÇÃO}

Nas últimas décadas, o volume de recursos repassados pela União para as 27 Unidades da Federação (UF) vem assinalando acelerado crescimento (Moutinho, 2016; Trompieri-Neto, Lopes, Barbosa, \& Holanda, 2009), o que requer a sua adequada alocação, para que se ofertem, de modo eficiente, os melhores benefícios à população. Em contrapartida, Afonso, Romero e Monsalve (2013) apontam que o gestor público enfrenta séria dificuldade ao decidir onde aplicar esses recursos de modo a maximizar o bem-estar social. Nesse contexto, Machado,
Irffi e Benegas (2011) entendem que a eficiência é um indicador fundamental na tomada de decisão na esfera pública.

Além dos repasses oriundos do governo federal, a arrecadação de tributos estaduais e municipais tem grande representatividade na composição dos recursos públicos dos estados. Dessa forma, a receita pública é obtida por meio da contribuição de toda a sociedade (Costa, Ferreira, Braga, \& Abrantes, 2015). Quanto à finalidade das receitas obtidas pela esfera pública, Beuren, Moura e Kloeppel (2013) esclarecem que elas têm por finalida- 
de cobrir gastos com o objetivo de atender às necessidades e demandas da sociedade. Assim, mostra-se importante a mensuração da eficiência no uso desses recursos, bem como do nível de qualidade de vida da população, por meio de indicadores socioeconômicos (Moura \& Sauer, 2009).

Além disso, argumenta-se que a mensuração da eficiência no setor público é relevante no contexto de países marcados pela intensa utilização do modelo burocrático na gestão pública, que, muitas vezes, tende ao desperdício de recursos (Balaguer-Coll, Prior \& Tortosa-Ausina, 2007; Secchi, 2009). Alinhado a isso, ressalta-se que, apesar de muitos países latino-americanos, como é o caso do Brasil, oscilarem entre diferentes concepções ideológicas, o modelo burocrático no setor púbico ainda prevalece (Santos \& Schommer, 2018), sinalizando a importância de investigar aspectos da gestão pública voltados à eficiência da utilização de recursos públicos, que é uma crítica frequente ao modelo burocrático (Secchi, 2009).

Entende-se como eficiência a utilização ótima dos recursos disponíveis, de modo a gerar o máximo de produtos ou serviços possíveis, ou seja, a eficiência está voltada à capacidade de executar algo, minimizando a relação entre insumo e produto (Peña, 2008; Rodrigues, 2017; Souza, Scatena, \& Kehrig, 2017). No âmbito da administração pública, compreende-se que a principal finalidade dos governos é oferecer bens e serviços públicos, com o intuito de ampliar os níveis de bem-estar social da população (Chiechelski, 2005; Debnath \& Shankar, 2014; Mukokoma \& Dijk, 2013).

Durante muito tempo, a renda nacional foi a medida mais comumente usada para se mensurar o nível de bem-estar social, sendo usualmente expressa pelo Produto Interno Bruto (PIB). Esse critério, porém, passou a sofrer críticas, entre elas o fato de que conhecer o PIB, ou seja, a renda de determinado país ou região, é insuficiente para se avaliar o grau de bem-estar da sociedade. Assim, no decorrer dos anos, a Organização das Nações Unidas (ONU) passou a trabaIhar na elaboração de um indicador capaz de expressar outras perspectivas de bem-estar social, surgindo, então, o Índice de Desenvolvimento Humano (IDH) (Scarpin \& Slomski, 2007).

Assim como o IDH, outros indicadores têm a característica de mensurar o desenvolvimento socioeconômico de determinado local, levando-se em conta diferentes dimensões de desenvolvimento, como o Índice FIRJAN de Desenvolvimento Municipal (IFDM) (Leite \& Fialho, 2015). Este estudo utiliza o IFDM, que, segundo Avelino, Bressan e Cunha (2013), surgiu a partir da necessidade de se monitorar o desenvolvimento socioeconômico anual dos municípios. O IFDM contempla três dimensões: emprego e renda, educação e saúde.

Destarte, compreende-se que a combinação entre o PIB e um indicador multidimensional de desenvolvimento, como o IDH e o IFDM, é capaz de expressar de maneira mais robusta o grau de bem-estar social, haja vista que estes, combinados, levam em conta não apenas aspectos econômicos, mas também aspectos relacionados a educação, saúde, saneamento e outros segmentos. No que tange à responsabilidade das UFs, estas devem estar aptas a alocar com eficiência os recursos disponíveis, de modo a propor- 
cionar à população altos níveis de qualidade em educação, saúde, saneamento, lazer e cultura, bem como colaborar no fortalecimento da economia, gerando, consequentemente, maiores indicadores de PIB e IDH (Scarpin \& Slomski, 2007).

Faé, Goulart e Abdala (2016) discutem acerca do papel do governo federal em relação ao desenvolvimento econômico e social da nação. Os autores ressaltam que, após a eleição do presidente Lula, em 2002, foram inseridos, na agenda de governo, alguns temas sobre o desenvolvimento socioeconômico do País, destacando-se a extinção da fome e da miséria e a ampliação do acesso à educação e à moradia. Essas bandeiras levantadas pelo então presidente foram mantidas após a eleição de sua sucessora, Dilma Vana Rousseff. Logo, percebe-se que, durante os governos Lula e Dilma, ganharam destaque o desenvolvimento socioeconômico e a promoção de políticas sociais. Adicionalmente, Pinto e Santos (2017) assinalam avanços em termos da participação de atores sociais nas decisões da administração pública federal nos governos Lula e Dilma, com destaque no governo Lula.

Considerando que a eficiência no setor público está relacionada à utilização dos recursos públicos a fim de ampliar o bem-estar social (Chiechelski, 2005) e que os governos dos ex-presidentes Lula e Dilma apresentaram, como principais bandeiras, o combate à pobreza extrema, ampliação e universalização do acesso à educação básica e saúde, que são condições básicas para a dignidade humana (Faé et al., 2016), além da busca pela inclusão social de grupos historicamente excluídos (Brito, 2017), este estudo levanta o seguinte questionamen- to: Quais UFs apresentaram os níveis mais altos de eficiência na alocação de recursos públicos durante os governos Lula e Dilma?

O estudo objetiva analisar a eficiência na alocação de recursos públicos nas 27 UFs durante os governos Lula e Dilma. Foram utilizados o IFDM, como indicador de bem-estar social, e o PIB, como indicador econômico (Avelino et al., 2013; Beuren et al. 2013; Scarpin \& Slomski, 2007). Foram combinados os indicadores IFDM e PIB, para se aquilatar a eficiência dos gastos públicos em relação ao bem-estar social e ao desenvolvimento socioeconômico nas 27 UFs.

Utiliza-se a Análise Envoltória de Dados (DEA) para analisar o grau de eficiência de cada UF, sendo cada uma delas utilizada no modelo como uma Unidade Tomadora de Decisão (Decision Making Unit - DMU), responsável por utilizar recursos (inputs) em prol da obtenção de produtos (outputs). Peña (2008) explica que a DEA vem sendo amplamente utilizada em estudos sobre a eficiência nos gastos públicos.

A eficiência dos gastos públicos tem sido objeto de várias investigações. Algumas delas dão ênfase à eficiência das finanças públicas e dos gastos por função (Costa et al., 2015; Diniz, Macedo, \& Corrar, 2012; Silva, Oliveira, Martins, \& Silva, 2014), enquanto outras concentram-se na eficiência dos gastos com educação (Scarpin, Macedo, Starosky, \& Rodrigues, 2012), outras em saúde (Souza \& Barros, 2013), e ainda outras na eficiência da governança eletrônica e sua correlação com a eficiência na utilização das receitas públicas (Beuren et al., 2013). Este estudo diferencia-se dos demais na medida em que investiga a eficiência dos gastos pú- 


\section{EFICIÊNCIA DA ALOCAÇÃO DE RECURSOS PÚBLICOS NAS UNIDADES DA FEDERAÇÃO NOS GOVERNOS LULA E DILMA}

blicos com educação e saúde, combinados com a renda, relacionando-os aos indicadores socioeconômico (PIB) e de qualidade de vida (IFDM).

Destaca-se ainda que analisar a eficiência da alocação dos recursos públicos agrega ao campo dos estudos sobre políticas públicas, uma vez que é papel do Estado se empenhar na aplicação de recursos, com foco no delineamento e implementação de políticas públicas que visem a oferta de bens e serviços públicos com o intuito de ampliar o bem-estar social e a qualidade de vida da sociedade, resultando na elevação dos índices de desenvolvimento socioeconômico (Chiechelski, 2005; Debnath \& Shankar, 2014).

\section{REVISÃO DA LITERATURA}

A definição dos objetivos das entidades e dos órgãos públicos deve guardar estreita consonância com seus deveres constitucionais e os anseios da sociedade (Silva et al., 2014). Para o alcance desses objetivos, faz-se necessária a gestão eficiente dos recursos públicos. No âmbito estadual, os recursos públicos advêm tanto de repasses federais quanto da arrecadação realizada pelos estados e municípios, e têm como principal função suprir as demandas sociais, proporcionando serviços que elevem o nível de qualidade de vida dos cidadãos. Moutinho (2016) afirma que o repasse de recursos públicos da União para estados e municípios é crescente, e que isso é previsto em diversos dispositivos legais, destacando-se as seguintes transferências: constitucionais, legais, diretas aos cidadãos, diretas ao Sistema Único de Saúde (SUS) e voluntárias.
Costa et al. (2015) argumentam que a gestão pública deve garantir mais acesso aos bens e serviços por parte da população, haja vista que os tributos são utilizados para financiar os gastos públicos. Os autores enfatizam, ainda, que os tributos são oriundos do esforço da sociedade, razão pela qual se espera que os gastos públicos sejam realizados de modo eficiente, garantindo o aumento do bem-estar daqueles que contribuíram para a composição dessas receitas. Esse bem-estar social vem sendo mensurado por meio de diferentes indicadores. Beuren et al. (2013) afirmam que o PIB e o $\mathrm{IDH}$, combinados, são indicadores capazes de dar uma ampla visão acerca da situação socioeconômica de municípios, estados e regiões, refletindo os respectivos níveis de bem-estar social.

Além do IDH, surgem outros indicadores com o propósito de mensurar o desenvolvimento socioeconômico de determinado local, considerando diferentes dimensões. $O$ IFDM - utilizado neste estudo - monitora o desenvolvimento socioeconômico dos municípios brasileiros (Avelino et al., 2013; Leite \& Fialho, 2015), levando em conta bases estatísticas públicas oficiais, e considera três diferentes dimensões: emprego e renda, educação e saúde (Federação das Indústrias do Estado do Rio de Janeiro [FIRJAN], 2015).

O PIB corresponde ao valor agregado de todos os bens e serviços finais produzidos dentro do território econômico de um país, independentemente da nacionalidade dos proprietários das unidades produtoras desses bens e prestadoras desses serviços (Sandroni, 1989). Trata-se, portanto, de um indicador unidimensional, razão pela qual 
tem sido criticada a sua capacidade de mensurar a qualidade de vida de toda uma sociedade (Moura \& Sauer, 2009).

Apesar das críticas ao uso do PIB, destaca-se sua relevância quanto à essência de seu objetivo principal, qual seja servir como indicador de desenvolvimento econômico. $\mathrm{O}$ PIB detém o papel de indicador-chave, haja vista que representa o credo estratégico das agências e das políticas locais, regionais e nacionais de desenvolvimento (Siedenberg, 2003). Partindo do pressuposto de que há necessidade de serem produzidos mais bens e prestados mais serviços para satisfazer as necessidades da humanidade, e que esse objetivo não será alcançado exclusivamente pela distribuição de bens e serviços públicos, o $\mathrm{PIB}$ reflete aspectos de desenvolvimento econômico necessários em uma análise multidimensional de qualidade de vida (Moura \& Sauer, 2009; Sandroni, 1989). Dessa forma, a despeito de algumas críticas e limitações em relação ao PIB, optou-se por utilizá-lo, já que se trata de uma medida capaz de refletir o desenvolvimento econômico em uma ampla perspectiva.

Quanto aos indicadores multidimensionais de desenvolvimento, o IDH e o IFDM apresentam como uma de suas maiores vantagens a capacidade de expressar, por meio de um único indicador, aspectos multidimensionais relativos à qualidade de vida (Leite \& Fialho, 2015; Moura \& Sauer, 2009). A qualidade de vida da sociedade, medida por tais indicadores de desenvolvimento, está relacionada com o uso eficiente dos recursos públicos (Debnath \& Shankar, 2014; Mukokoma \& Dijk, 2013). Considerando-se que o conceito de eficiência é frequente- mente abordado quando se faz alusão a gastos públicos, vale enfatizar que o termo compreende a combinação ótima da utilização de recursos com os métodos adotados em um processo de produção, gerando o máximo possível (Peña, 2008; Souza et al., 2017). Logo, a eficiência, para os fins deste estudo, visa otimizar os recursos, garantindo o meIhor resultado por meio da utilização mínima de recursos, sem comprometer a qualidade do produto final (Banker \& Natarajan, 2008; Hauner \& Kyobe, 2010; Peña, 2008).

A eficiência na gestão pública está voltada para a capacidade que tem o Estado de prover bens e serviços com o intuito de promover o bem-estar social (Chiechelski, 2005). Scarpin et al. (2012) apontam que, devido à escassez dos recursos públicos, os gastos devem ser feitos de modo eficiente, visando atender às demandas sociais. Logo, a eficiência é um valor intrínseco da administração pública. Além disso, destaca-se o princípio da eficiência dos gastos públicos, que versa acerca do papel da administração pública ao utilizar os recursos de maneira idônea, econômica e satisfatória (Silva et al., 2014).

Considerando o objetivo deste estudo de analisar o nível de eficiência das 27 UFs quanto à alocação de gastos públicos, utiliza-se a DEA, que, segundo Peña (2008), tem sido aplicada com sucesso em pesquisas sobre eficiência no campo da gestão pública. Trata-se de um método não paramétrico, desenvolvido por Charnes et al. (1978), que determina a curva de eficiência por meio de programação matemática de otimização, analisando o desempenho relativo de diferentes Unidades Tomadoras de Decisão (Decision Making Units - DMU) quanto à utilização dos mesmos tipos de recursos 


\section{EFICIÊNCIA DA ALOCAÇÃO DE RECURSOS PÚBLICOS NAS UNIDADES DA FEDERAÇÃO NOS GOVERNOS LULA E DILMA}

para produzir idênticos produtos. A seguir, apresentam-se alguns estudos que utilizaram a DEA para medir a eficiência no setor público.

Diniz et al. (2012) avaliaram a eficiência financeira na gestão dos recursos públicos de municípios e mensuraram sua relação com os gastos nas funções de governo definidas na estrutura orçamentária brasileira. Os autores analisaram os dados de municípios com população superior a 200 mil habitantes e calcularam a eficiência financeira dos municípios por meio do método DEA, utilizando as variáveis despesas fixas, despesas com pessoal, dívida consolidada, dívida a curto prazo e necessidade de recursos, como inputs, e as variáveis receita per capita, participação de receitas próprias, importância do excedente acumulado, relação das receitas e despesas totais e índice de investimento, como outputs. Em seguida, os autores conduziram uma regressão linear para investigar as relações entre as variáveis. Os resultados indicaram que, das 28 funções de governo dispostas na execução orçamentária, somente os gastos per capita com saúde, urbanismo e saneamento apresentaram efeito sobre a eficiência financeira.

Scarpin et al. (2012) analisaram a eficiência dos recursos públicos direcionados para a educação em 285 municípios de Santa Catarina. A partir da aplicação da DEA em dados obtidos no Finanças Brasil (Finbra) e no Instituto Brasileiro de Geografia e Estatística (IBGE), os resultados apontaram que $12 \%$ dos municípios catarinenses são eficientes em seus gastos com educação e que os menos populosos tendem a ser os mais eficientes.
Beuren et al. (2013) examinaram as práticas de governança eletrônica dos estados brasileiros e sua correlação com a eficiência na utilização das receitas totais. A partir da DEA, os resultados mostram que todas as UFs do Sudeste e do Sul podem ser consideradas mais eficientes que as das outras três regiões. Os achados evidenciam, ainda, que as UFs com melhores práticas de governança eletrônica são as que apresentam mais eficiência na utilização das receitas.

Souza e Barros (2013) analisaram a eficiência dos gastos públicos com assistência hospitalar em 23 UFs em 2009 e 2010. Os resultados revelaram que seis (Acre, Amapá, Paraná, Roraima, São Paulo e Tocantins) foram considerados eficientes pela observação de seus escores em 2009. Em 2010, a eficiência foi visualizada em sete UFs (Acre, Amapá, Minas Gerais, Paraná, Roraima, São Paulo e Tocantins).

Silva et al. (2014) avaliaram se as 27 UFs foram eficientes na aplicação das despesas totais de investimentos per capita apuradas no período de 2003 a 2012. Utilizada a DEA, os resultados indicaram que o Acre foi a única UF considerada eficiente, nos modelos de Retornos Constantes à Escala, Retornos Variáveis à Escala e de Escala com orientação a output. No modelo Retornos Variáveis à Escala, além do Acre, foram considerados eficientes o Ceará, o Espírito Santo, o Maranhão e o Piauí.

Costa et al. (2015) investigaram os fatores associados aos níveis de eficiência na alocação de recursos públicos em 831 municípios de Minas Gerais. Com a aplicação da DEA e da análise de regressão, verificaram-se os níveis de eficiência na alocação de 
recursos públicos com vistas à promoção do desenvolvimento socioeconômico. Constatou-se que os municípios com as piores condições socioeconômicas têm sua gestão pública influenciada por uma gama maior de variáveis exógenas.

\section{PROCEDIMENTOS METODOLÓGICOS}

A população da pesquisa compreende as 27 UFs. Em relação ao período analisado, foram considerados os três primeiros anos do segundo mandato do presidente Lula e os três primeiros do primeiro mandato da presidente Dilma, haja vista que a Lei Complementar $\mathrm{n}$ - 101, de 4 de maio de 2000 (2000, Lei de Responsabilidade Fiscal), impõe limites de gastos em anos eleitorais, de modo que a sua inclusão poderia vir a comprometer a análise.

A pesquisa faz uso da DEA, método não paramétrico, desenvolvido por Charnes et al. (1978), amplamente utilizado em estudos relacionados a eficiência, que determina a curva de eficiência por meio de programação matemática de otimização, sendo também conhecido como modelo CCR, numa alusão às iniciais dos autores. Esse modelo analisa o desempenho relativo de diferentes Decision Making Units (DMU) na utilização dos mesmos tipos de recursos para produzir idênticos produtos.

A DEA calcula a eficiência técnica das DMUs na razão (virtual) entre os produtos (outputs) e os insumos (inputs), utilizando programação matemática linear para estimar a fronteira da possibilidade de produção. O resultado obtido por meio do modelo varia entre 0 e 1 , em que " 0 " indica que a
DMU é ineficiente, e "1" significa que a DMU é eficiente.

A modalidade CCR foi elaborada com o intuito de analisar retornos constantes de escala (Constant Returns to Scale - CRS), em que a fronteira eficiente é representada por uma reta, indo da origem até a unidade produtiva que forma o maior raio com o eixo do insumo. A modalidade de DEA desenvolvida por Banker et al. (1984), conhecida como BCC, igualmente em alusão às iniciais dos nomes dos seus proponentes, diferencia-se da modalidade CCR por incluir retornos variáveis de escala (Variable Returns to Scale - VRS), em que uma fronteira convexa eficiente é formada com as melhores unidades, independentemente da escala de operação. Assim, a modalidade BCC permite que a eficiência máxima sofra variações em função da economia de escala, possibilitando comparar unidades de diferentes portes (Peña, 2008).

Inicialmente, calculou-se a eficiência dos gastos das 27 UFs considerando-se como produto a geração de bem-estar social, expresso pelos respectivos IFDMs. Utilizou-se a DEA nas modalidades CCR e BCC, com orientação de output para o cálculo das eficiências. Posteriormente, calcularam-se os coeficientes de correlação de Pearson e de Spearman, para se verificarem os níveis de correlação entre a eficiência dos gastos públicos na geração de bem-estar social de cada UF nas modalidades CCR e BCC e o respectivo PIB. Também foram feitos testes de correlação entre as duas modalidades da DEA.

O Quadro 1 traz as DMUs (representadas pelas 27 UFs) para a aplicação na DEA. 
EFICIÊNCIA DA ALOCAÇÃo dE RECURSOS PÚBLICOS NAS UNIDADES DA FEDERAÇÃo NOS GOVERNOS LULA E DILMA

Quadro 1. DMUs utilizadas na pesquisa e UFs correspondentes

\begin{tabular}{|c|c|c|c|c|c|}
\hline $\mathrm{N}^{\circ}$ & UF (sigla) & $\mathrm{N}^{\circ}$ & UF (sigla) & $\mathrm{N}^{\circ}$ & UF (sigla) \\
\hline 1 & $\mathrm{AC}$ & 10 & $\mathrm{MA}$ & 19 & $\mathrm{RJ}$ \\
\hline 2 & $\mathrm{AL}$ & 11 & $\mathrm{MG}$ & 20 & $\mathrm{RN}$ \\
\hline 3 & $\mathrm{AM}$ & 12 & $\mathrm{MS}$ & 21 & $\mathrm{RO}$ \\
\hline 4 & $\mathrm{AP}$ & 13 & $\mathrm{MT}$ & 22 & $\mathrm{RR}$ \\
\hline 5 & $\mathrm{BA}$ & 14 & $\mathrm{PA}$ & 23 & $\mathrm{RS}$ \\
\hline 6 & $\mathrm{CE}$ & 15 & $\mathrm{~PB}$ & 24 & $\mathrm{SC}$ \\
\hline 7 & $\mathrm{DF}$ & 16 & $\mathrm{PE}$ & 25 & $\mathrm{SE}$ \\
\hline 8 & $\mathrm{ES}$ & 17 & $\mathrm{PI}$ & 26 & $\mathrm{SP}$ \\
\hline 9 & $\mathrm{GO}$ & 18 & $\mathrm{PR}$ & 27 & $\mathrm{TO}$ \\
\hline
\end{tabular}

A escolha das variáveis de input deu-se pelo fato de que a Constituição da República Federativa do Brasil de 1988 (1988) prevê gastos mínimos com áreas prioritárias como a educação e a saúde, além de destacar que essas são áreas fundamentais e básicas para a dignidade humana. Outra variável de input utilizada foi a renda domiciliar per capita, uma vez que a renda é um dos potencializadores do desenvolvimento social e econômico (Morais, 2015). Como output, optou-se pela média estadual do IFDM, que é um indicador multidimensional de desenvolvimento socioeconômico, ou seja, um produto obtido pela utilização dos gastos com educação e saúde e pela geração de renda das UFs.
Quanto às variáveis de inputs e outputs aplicadas na DEA, o input 1 (gastos com educação) e o input 2 (gastos com saúde) foram extraídos do website da Secretaria do Tesouro Nacional (STN) (2016), o input 3 (renda domiciliar per capita) foi obtido no website do Instituto Brasileiro de Geografia e Estatística (IBGE) (2016), e o output 1 (IFDM) foi obtido no website da FIRJAN (2016). Como o IFDM é um indicador municipal, foi calculada a média para cada UF, a partir dos indicadores dos respectivos municípios. O Quadro 2 mostra um resumo das variáveis e suas respectivas fontes de coleta.

Quadro 2. Definição das variáveis para a DEA

\begin{tabular}{|l|l|c|}
\hline & \multicolumn{1}{|c|}{ Variável } & Fonte \\
\hline Input 1 & Gasto com educação & STN \\
\hline Input 2 & Gasto com saúde & STN \\
\hline Input 3 & Renda domiciliar per capita & IBGE \\
\hline Output 1 & IFDM & FIRJAN \\
\hline
\end{tabular}


Destaca-se que os valores monetários absolutos foram reduzidos, por meio da conversão em logaritmo natural, para viabilizar a melhor operacionalização das variáveis. Assim, os gastos com educação são representados por LogGE, os gastos com saúde são representados por LogGS, e a renda domiciliar per capita é representada por LogRD. Os valores do IFDM não foram afetados, haja vista que já são expressos como um indicador, com valor entre 0 e 1 .

Após o cálculo da eficiência dos gastos públicos na geração de bem-estar social, para cada UF durante os anos analisados, calcularam-se os coeficientes de correlação de Pearson e de Spearman entre o valor obtido por meio da DEA de cada UF, nas modalidades CCR e BCC, e o PIB respectivo. Os valores do PIB também foram convertidos em logaritmo natural (LogPIB), adotando-se o mesmo tratamento das outras variáveis monetárias.

Após identificar-se a normalidade dos da- dos, optou-se pelo teste de correlação mais apropriado para cada tipo de dados - teste de Pearson para dados paramétricos e teste de Spearman para dados não paramétricos. Ao fim dessas análises, pôde-se identificar o coeficiente de correlação e o grau de significância, para que, assim, fosse feita alguma inferência acerca dos dados.

Utilizou-se o Sistema Integrado de Apoio à Decisão (Siad), na versão 3.0 (Meza, Biondi, Mello, \& Gomes, 2005), para os cálculos da DEA nas modalidades CCR e BCC, assim como o aplicativo Stata, na versão 20.0, para os testes de normalidade e de correlação de Pearson e Spearman.

\section{ANÁLISE DOS DADOS}

Inicialmente, realizou-se uma análise descritiva das variáveis, a fim de se verificar o seu comportamento. A Tabela 1 mostra os valores mínimo e máximo, a média, o desvio-padrão e o coeficiente de variação correspondentes a cada variável.

Tabela 1. Estatística descritiva das variáveis da pesquisa

\begin{tabular}{cccccc}
\hline Variável & Mínimo & Máximo & Média & Desvio-padrão & $\begin{array}{c}\text { Coeficiente de } \\
\text { variação }\end{array}$ \\
\hline LogGS & 8,2924 & 10,2646 & 9,1099 & 0,3825 & 0,0420 \\
LogGE & 8,4595 & 10,4830 & 9,2090 & 0,4055 & 0,0440 \\
LogRD & 2,6209 & 3,3084 & 2,8946 & 0,1497 & 0,0517 \\
IFDM & 0,4066 & 0,7956 & 0,5871 & 0,0970 & 0,1653 \\
LogPIB & 9,6200 & 12,2325 & 10,8040 & 0,5534 & 0,0512 \\
\hline
\end{tabular}


Observa-se que o IFDM é a variável que apresenta o menor desvio-padrão, enquanto o PIB assinala o maior desvio-padrão. Nota-se uma maior dispersão nos valores do PIB. Evidencia-se que os dados apresentam homogeneidade, hajam vista os baixos valores dos coeficientes de variação.
Para uma percepção mais aprofundada das variáveis, aplicou-se o teste de diferenças entre médias, em que se verificaram as variáveis utilizadas na DEA a partir da mediana dos valores do PIB das 27 UFs. A Tabela 2 apresenta os resultados referentes ao teste de diferenças entre médias.

Tabela 2. Teste de diferenças entre médias do PIB e as variáveis utilizadas nas duas modalidades da DEA

\begin{tabular}{cccccc}
\hline \multirow{2}{*}{ Variável } & Grupos de & Número de & Média & $Z$ & p-value \\
\hline \multirow{2}{*}{ LogGS } & UFs & observações & & & \\
& Menor PIB & 81 & 9,3985 & $-10,415$ & 0,0000 \\
& Maior PIB & 81 & 8,8213 & & \\
LogGE & Menor PIB & 81 & 9,5066 & $-10,308$ & 0,0000 \\
& Maior PIB & 81 & 8,9114 & & \multirow{2}{*}{0,0000} \\
\multirow{2}{*}{ LogRD } & Menor PIB & 81 & 2,9769 & $-6,683$ & \\
& Maior PIB & 81 & 0,6417 & & 0,0000 \\
\hline
\end{tabular}

Com base na Tabela 2, rejeita-se a hipótese nula de igualdade entre as médias para todas as variáveis utilizadas na DEA. Assim, nota-se uma significância ao nível de $1 \%$ para as UFs com maior e menor PIB em relação às demais variáveis, ou seja, as UFs com PIB mais alto apresentam maiores gas- tos com educação e saúde, maior renda familiar e IFDM também maior.

A Tabela 3 traz os escores de eficiência obtidos por meio da DEA nas modalidades CCR e BCC de cada UF durante os governos Lula e Dilma. 
Tabela 3. Resultado da eficiência calculada pela DEA nas modalidades CCR e BCC, com orientação de output, das 27 UFs nos governos Lula e Dilma

\begin{tabular}{|c|c|c|c|c|c|c|c|c|c|c|c|c|}
\hline \multirow{3}{*}{ UF) } & \multicolumn{6}{|c|}{ Governo Lula } & \multicolumn{6}{|c|}{ Governo Dilma } \\
\hline & \multicolumn{2}{|c|}{2007} & \multicolumn{2}{|c|}{2008} & \multicolumn{2}{|c|}{2009} & \multicolumn{2}{|c|}{2011} & \multicolumn{2}{|c|}{2012} & \multicolumn{2}{|c|}{2013} \\
\hline & CCR & $\mathrm{BCC}$ & CCR & $\mathrm{BCC}$ & CCR & $\mathrm{BCC}$ & CCR & $\mathrm{BCC}$ & CCR & $\mathrm{BCC}$ & CCR & $\mathrm{BCC}$ \\
\hline$A C$ & 0,716 & 0,910 & 0,695 & 0,903 & 0,692 & 0,843 & 0,738 & 0,901 & 0,738 & 0,879 & 0,752 & 0,922 \\
\hline $\mathrm{AL}$ & 0,709 & 1,000 & 0,727 & 1,000 & 0,752 & 1,000 & 0,807 & 1,000 & 0,816 & 1,000 & 0,819 & 1,000 \\
\hline AM & 0,633 & 0,739 & 0,609 & 0,723 & 0,647 & 0,757 & 0,670 & 0,745 & 0,669 & 0,742 & 0,676 & 0,709 \\
\hline AP & 0,739 & 0,947 & 0,738 & 1,000 & 0,728 & 0,979 & 0,769 & 1,000 & 0,785 & 0,972 & 0,792 & 0,980 \\
\hline BA & 0,639 & 0,729 & 0,639 & 0,739 & 0,667 & 0,744 & 0,712 & 0,766 & 0,727 & 0,786 & 0,737 & 0,770 \\
\hline CE & 0,864 & 1,000 & 0,856 & 1,000 & 0,887 & 1,000 & 0,920 & 1,000 & 0,919 & 1,000 & 0,940 & 1,000 \\
\hline DF & 1,000 & 1,000 & 1,000 & 1,000 & 1,000 & 1,000 & 1,000 & 1,000 & 1,000 & 1,000 & 1,000 & 1,000 \\
\hline ES & 0,940 & 1,000 & 0,924 & 1,000 & 0,946 & 1,000 & 1,000 & 1,000 & 1,000 & 1,000 & 1,000 & 1,000 \\
\hline GO & 0,892 & 0,946 & 0,870 & 0,938 & 0,892 & 0,942 & 0,921 & 0,927 & 0,926 & 0,936 & 0,946 & 0,947 \\
\hline MA & 0,689 & 1,000 & 0,701 & 1,000 & 0,711 & 0,926 & 0,748 & 1,000 & 0,735 & 0,846 & 0,746 & 0,877 \\
\hline MG & 0,871 & 0,925 & 0,830 & 0,889 & 0,859 & 0,901 & 0,900 & 0,919 & 0,910 & 0,931 & 0,923 & 0,931 \\
\hline MS & 0,888 & 1,000 & 0,863 & 1,000 & 0,890 & 1,000 & 0,912 & 1,000 & 0,926 & 1,000 & 0,954 & 1,000 \\
\hline MT & 0,874 & 0,963 & 0,834 & 0,932 & 0,883 & 0,974 & 0,925 & 0,999 & 0,930 & 0,973 & 0,942 & 0,964 \\
\hline PA & 0,654 & 0,759 & 0,649 & 0,774 & 0,677 & 0,805 & 0,707 & 0,784 & 0,731 & 0,809 & 0,736 & 0,795 \\
\hline PB & 0,755 & 0,899 & 0,741 & 0,893 & 0,765 & 0,906 & 0,804 & 0,917 & 0,830 & 0,951 & 0,850 & 0,972 \\
\hline $\mathrm{PE}$ & 0,791 & 0,941 & 0,786 & 0,937 & 0,822 & 0,959 & 0,864 & 0,957 & 0,862 & 0,932 & 0,879 & 0,927 \\
\hline $\mathrm{PI}$ & 0,694 & 0,872 & 0,724 & 1,000 & 0,744 & 0,926 & 0,790 & 0,958 & 0,788 & 0,929 & 0,802 & 0,931 \\
\hline PR & 0,932 & 0,965 & 0,903 & 0,950 & 0,929 & 0,964 & 0,957 & 0,961 & 0,959 & 0,964 & 0,973 & 0,975 \\
\hline RJ & 0,916 & 0,940 & 0,879 & 0,911 & 0,893 & 0,913 & 0,940 & 0,943 & 0,954 & 0,958 & 0,957 & 0,961 \\
\hline $\mathrm{RN}$ & 0,819 & 0,948 & 0,806 & 0,951 & 0,829 & 0,966 & 0,857 & 0,948 & 0,862 & 0,947 & 0,875 & 0,964 \\
\hline RO & 0,782 & 0,957 & 0,768 & 0,968 & 0,792 & 0,951 & 0,850 & 0,991 & 0,860 & 0,995 & 0,876 & 1,000 \\
\hline $\mathrm{RR}$ & 0,715 & 1,000 & 0,689 & 1,000 & 0,732 & 1,000 & 0,759 & 1,000 & 0,795 & 1,000 & 0,782 & 1,000 \\
\hline RS & 0,927 & 0,963 & 0,896 & 0,938 & 0,919 & 0,948 & 0,952 & 0,954 & 0,954 & 0,958 & 0,964 & 0,972 \\
\hline SC & 0,933 & 0,966 & 0,907 & 0,973 & 0,922 & 0,949 & 0,986 & 0,987 & 0,989 & 0,992 & 1,000 & 1,000 \\
\hline SE & 0,823 & 1,000 & 0,815 & 1,000 & 0,827 & 1,000 & 0,867 & 0,999 & 0,872 & 0,992 & 0,856 & 1,000 \\
\hline $\mathrm{SP}$ & 0,996 & 1,000 & 0,985 & 1,000 & 0,989 & 1,000 & 1,000 & 1,000 & 1,000 & 1,000 & 1,000 & 1,000 \\
\hline TO & 0,847 & 1,000 & 0,812 & 0,984 & 0,828 & 0,994 & 0,870 & 0,983 & 0,881 & 0,984 & 0,887 & 1,000 \\
\hline
\end{tabular}

Para se analisarem de modo mais pontual os dados da Tabela 3, a Tabela 4 mostra o ranking das cinco UFs mais eficientes e o das cinco menos eficientes, durante os anos analisados dos governos Lula e Dilma, tomando-se por base os resultados da DEA na modalidade CCR. 
EFICIÊNCIA DA ALOCAÇÃO DE RECURSOS PÚBLICOS NAS UNIDADES DA FEDERAÇÃo NOS GOVERNOS LULA E DILMA

Tabela 4. Ranking das UFs mais e menos eficientes nos governos Lula e Dilma, com base na DEA modalidade CCR

\begin{tabular}{|c|c|c|c|c|c|c|c|c|c|c|c|c|}
\hline \multirow{3}{*}{ Posição } & \multicolumn{6}{|c|}{ Governo Lula } & \multicolumn{6}{|c|}{ Governo Dilma } \\
\hline & \multicolumn{2}{|c|}{2007} & \multicolumn{2}{|c|}{2008} & \multicolumn{2}{|c|}{2009} & \multicolumn{2}{|c|}{2011} & \multicolumn{2}{|c|}{2012} & \multicolumn{2}{|c|}{2013} \\
\hline & UF & CCR & UF & CCR & UF & CCR & UF & CCR & UF & CCR & UF & CCR \\
\hline \multicolumn{13}{|c|}{ UFs mais eficientes } \\
\hline $1^{a}$ & DF & 1,0000 & DF & 1,0000 & DF & 1,0000 & DF & 1,0000 & DF & 1,0000 & DF & 1,0000 \\
\hline $2^{\mathrm{a}}$ & SP & 0,9955 & SP & 0,9849 & SP & 0,9890 & ES & 1,0000 & ES & 1,0000 & ES & 1,0000 \\
\hline $3^{a}$ & ES & 0,9396 & ES & 0,9241 & ES & 0,9463 & SP & 1,0000 & $\mathrm{SP}$ & 1,0000 & SC & 1,0000 \\
\hline $4^{a}$ & SC & 0,9330 & SC & 0,9072 & PR & 0,9292 & SC & 0,9863 & SC & 0,9888 & SP & 1,0000 \\
\hline $5^{a}$ & PR & 0,9324 & PR & 0,9026 & SC & 0,9220 & PR & 0,9569 & PR & 0,9591 & PR & 0,9729 \\
\hline \multicolumn{13}{|c|}{ UFs menos eficientes } \\
\hline $23^{a}$ & $\mathrm{PI}$ & 0,6937 & $A C$ & 0,6947 & MA & 0,7112 & MA & 0,7475 & $A C$ & 0,7376 & $A C$ & 0,7517 \\
\hline $24^{a}$ & MA & 0,6894 & $\mathrm{RR}$ & 0,6886 & $A C$ & 0,6924 & $A C$ & 0,7381 & MA & 0,7346 & MA & 0,7456 \\
\hline $25^{\mathrm{a}}$ & PA & 0,6535 & $\mathrm{PA}$ & 0,6487 & PA & 0,6773 & PA & 0,7120 & PA & 0,7313 & BA & 0,7371 \\
\hline $26^{a}$ & $\mathrm{BA}$ & 0,6390 & $\mathrm{BA}$ & 0,6393 & $\mathrm{BA}$ & 0,6668 & BA & 0,7066 & $\mathrm{BA}$ & 0,7273 & $\mathrm{PA}$ & 0,7362 \\
\hline $27^{a}$ & AM & 0,6334 & AM & 0,6093 & AM & 0,6470 & AM & 0,6702 & AM & 0,6690 & AM & 0,6763 \\
\hline
\end{tabular}

No período do governo Lula, somente o Distrito Federal foi eficiente, sendo a única UF a apresentar escore 1, indicando o alcance da linha de eficiência do modelo durante o triênio considerado. Observa-se também que Espírito Santo e São Paulo se mantiveram na $3^{\underline{a}}$ e na $2^{\underline{a}}$ posição, respectivamente, durante o governo Lula. Além disso, Paraná e Santa Catarina também integram o ranking das UFs mais eficientes. Paraná ascende da $5^{\text {a }}$ posição, que ocupou em 2007 e 2008, para a $4^{\mathrm{a}}$ em 2009. Enquanto isso, Santa Catarina ocupou a $4^{a}$ posição em 2007 e 2008, descendo para a 5ª posição em 2009.

Acerca das UFs menos eficientes no governo Lula, nota-se que Amazonas, Bahia e Pará ocuparam a última, a penúltima e a antepenúltima posição do ranking, respectivamente, durante todo o período analisado. Enquanto isso, o Piauí ocupou a $23^{\text {a }}$ posição em 2007, seguido do Maranhão, em $24^{\circ}$ lugar. Em 2008, Acre e Roraima ocuparam a $23^{\text {a }}$ e a $24^{a}$ posição, respectivamente. Em 2009 , o Acre continuou entre os menos eficientes, passando da $23^{\underline{a}}$ para a $24^{\text {a }}$ posição, apontando um valor ainda menor em sua escala de eficiência, enquanto o Maranhão, que, em 2007, aparece como uma das cinco UFs menos eficientes, reaparece em 2009, ocupando a 23를 posição.

Quanto à eficiência das UFs no governo Dilma, nota-se que, em 2011 e 2012, o Distrito Federal, o Espírito Santo e São Paulo atingiram a linha de eficiência do modelo, obtendo escore 1 de eficiência. No mesmo biênio, Santa Catarina ocupou a $4^{a}$ posição do ranking, ao passo que, em 2013, obteve escore 1, juntando-se ao Distrito Federal, ao Espírito Santo e a São Paulo, que continuaram no ranking das UFs mais eficientes, com escore 1. O Paraná ocupou o 5 lugar durante todo o triênio analisado do governo Dilma. 
Analisando-se as UFs menos eficientes no governo Dilma, observa-se que Acre, Amazonas, Bahia, Maranhão e Pará aparecem nas cinco últimas posições do ranking durante todo o triênio considerado, apenas sofrendo alternância de posições. O Amazonas ocupou a última posição durante os três anos. O Acre e o Maranhão oscilaram entre a $23^{a}$ e a $24^{a}$ posição ao longo do período, enquanto a Bahia e o Pará se revezaram entre a $25^{\underline{a}}$ e a $26^{\mathrm{a}}$.

Percebe-se que, tanto no governo Lula quanto no governo Dilma, somente as UFs localizadas nas regiões Centro-Oeste, Sudeste e Sul ocuparam as cinco primeiras posições.
Em contrapartida, as UFs das regiões Nordeste e Norte figuram nas cinco últimas posições.

Dando-se continuidade à análise pontual dos dados da Tabela 3, optou-se por analisar as eficiências obtidas por meio do modelo DEA na modalidade BCC de maneira agrupada por região, pois, como esse modelo considera retornos variáveis de escala, tende a ser maior o número de unidades tomadoras de decisões eficientes. A Tabela 5 mostra a distribuição quantitativa das UFs eficientes por região, com base no modelo DEA na modalidade BCC.

Tabela 5. Distribuição anual das UFs eficientes, por região, baseada no modelo DEA na modalidade BCC - 2007-2013

\begin{tabular}{lcccccc}
\hline \multirow{2}{*}{ Região } & \multicolumn{3}{c}{ Governo Lula } & \multicolumn{3}{c}{ Governo Dilma } \\
\cline { 2 - 7 } & $\mathbf{2 0 0 7}$ & $\mathbf{2 0 0 8}$ & $\mathbf{2 0 0 9}$ & $\mathbf{2 0 1 1}$ & $\mathbf{2 0 1 2}$ & $\mathbf{2 0 1 3}$ \\
\hline Centro-Oeste & 2 & 2 & 2 & 2 & 2 & 2 \\
Nordeste & 4 & 5 & 3 & 3 & 2 & 3 \\
Norte & 2 & 2 & 1 & 2 & 1 & 3 \\
Sudeste & 2 & 2 & 2 & 2 & 2 & 2 \\
Sul & - & - & - & - & - & 1 \\
\multicolumn{1}{c}{ Total } & $\mathbf{1 0}$ & $\mathbf{1 1}$ & $\mathbf{8}$ & $\mathbf{9}$ & $\mathbf{7}$ & $\mathbf{1 1}$ \\
\hline
\end{tabular}

O modelo DEA, na modalidade BCC na análise da eficiência das UFs no governo Lula, indica que, em 2007, 10 UFs apresentam escore 1. Nesse mesmo ano, o Nordeste apresenta o maior número de UFs eficientes (quatro), enquanto o Sul não apresenta nenhuma. O Centro-Oeste, o Norte e o Sudeste apresentam duas UFs, cada, com escore de eficiência 1. Em 2008, são observadas 11 UFs eficientes, uma a mais em relação ao ano anterior, assim distribuídas: duas no Centro-Oeste, cinco no Nordeste, duas no Norte e duas no Sudeste - o Sul continua sem apresentar UFs eficientes para esse modelo. Em 2009, o número de UFs eficientes é reduzido a oito, sendo duas situadas no Centro-Oeste, três no Nordeste, uma no Norte e duas no Sudeste.

No governo Dilma, os resultados foram semelhantes aos de seu antecessor. Verifica-se que no Centro-Oeste e no Sudeste houve quatro UFs eficientes em todos os anos do governo Dilma, sendo duas em cada região. O Sul apresentou uma UF eficiente em 2013, porém nenhuma em 2011 e 2012. O Nordeste apresentou três, duas e três UFs eficientes em 2011, 2012 e 2013, respectivamente. E 
o Norte assinalou duas UFs eficientes em 2011, uma em 2012 e três em 2013.

Nota-se uma divergência entre os resultados da DEA obtidos nas modalidades CCR e BCC. Na BCC, somente UFs do Sudeste e do Sul apresentaram escore 1 de eficiência. Isso ocorreu porque a modalidade CCR obedece ao critério de proporcionalidade entre os inputs e outputs; ou seja, o volume de outputs obtidos deve ser proporcional ao número de inputs aplicados, gerando, assim, uma linha de eficiência. A modalidade BCC está relacionada à ideia de retornos variáveis de escala, substituindo o critério de proporcionalidade presente na modalidade CCR; ou seja, a modalidade BCC analisa a capacidade da unidade tomadora de decisão de utilizar os inputs de maneira mais econômica em prol da obtenção de outputs, atingindo, assim, a curva de eficiência.
Para se compreender a forma como a eficiência da alocação dos recursos públicos no desenvolvimento multidimensional das 27 UFs está relacionada à realidade econômica de cada uma delas, foram feitos testes de correlação entre os escores de eficiência obtidos por cada UF por meio da DEA nas modalidades CCR e BCC e o PIB respectivo. Também foram feitas correlações entre as duas modalidades da DEA.

Os testes de normalidade apontam que os dados são não paramétricos. Foram considerados os resultados do teste de Spearman, que foram semelhantes aos do teste de Pearson. A Tabela 6 traz os resultados das correlações entre as variáveis para o governo Lula, enquanto a Tabela 7 expõe os resultados do governo Dilma.

Tabela 6. Correlação de Spearman para as eficiências calculadas nas modalidades CCR e BCC e o PIB das 27 UFs no governo Lula

\begin{tabular}{lcl}
\hline Correlação & Coeficiente & Significância dos resultados \\
\hline CCR e BCC & $+0,4322$ & Correlação significativa ao nível de 0,01 \\
CCR e PIB & $+0,5888$ & Correlação significativa ao nível de 0,01 \\
BCC e PIB & $-0,1816$ & Correlação não significativa \\
\hline
\end{tabular}

Nota-se uma correlação positiva entre as modalidades CCR e BCC, com coeficiente 0,4322 , assim como uma correlação positiva entre CCR e PIB, com coeficiente 0,5888 . Os resultados apontam que não há significância entre a modalidade BCC e o PIB. Assim, quanto maior for a eficiência na modalidade CCR, maior será a eficiência na modalidade BCC. O mesmo ocorre em relação ao PIB e à modalidade CCR. 
Clayton Robson Moreira da Silva - Roberta Michele Ponte Alves - Márcia Martins Mendes De Luca - Alessandra Carvalho de Vasconcelos

Tabela 7. Correlação de Spearman para as eficiências calculadas nas modalidades CCR e BCC e o PIB das 27 UFs no governo Dilma

\begin{tabular}{ccl}
\hline Correlação & Coeficiente & Significância dos resultados \\
\hline CCR e BCC & $+0,5286$ & Correlação significativa ao nível de 0,01 \\
CCR e PIB & $+0,5955$ & Correlação significativa ao nível de 0,01 \\
BCC e PIB & $-0,1196$ & Correlação não significativa \\
\hline
\end{tabular}

Quanto às correlações no governo Dilma, nota-se, na Tabela 7, que, assim como no governo Lula, há uma correlação positiva entre as modalidades CCR e BCC, com coeficiente 0,5286 (superior ao do governo Lula), assim como há uma correlação positiva entre a modalidade CCR e o PIB, com coeficiente 0,5955 (superior ao do governo Lula). Não se pode inferir acerca da correlação entre as modalidades BCC e o PIB, pois não foi apresentada significância. Destarte, quanto maior for a eficiência na modalidade CCR, maior será a eficiência na modalidade BCC. O mesmo ocorre em relação ao PIB e à modalidade CCR.

\section{CONSIDERAÇÕES FINAIS}

O estudo analisou a eficiência na alocação de recursos públicos nas 27 UFs durante os governos Lula e Dilma. Utilizou-se a DEA para se calcular a eficiência da alocação dos gastos públicos nas 27 UFs. Como variáveis de entrada para o modelo, utilizaram-se os gastos com saúde, os gastos com educação e a renda familiar, e, como saída, o IFDM. As variáveis de entrada convergem com os aspectos multidimensionais do IFDM: educação, saúde e renda.

Constatou-se que, durante o governo Lula, pela DEA na modalidade CCR, somente o Distrito Federal mostrou-se eficiente na alocação dos gastos públicos. No governo Dilma, notou-se que o Distrito Federal, o
Espírito Santo, Santa Catarina e São Paulo passaram a integrar o ranking como UFs eficientes. $O$ ranking das UFs mais eficientes e menos eficientes apontou que as mais eficientes se localizam no Centro-Oeste, no Sudeste e no Sul, enquanto as menos eficientes localizam-se no Nordeste e no Norte.

Os resultados das eficiências pela DEA na modalidade BCC indicaram que, no governo Lula, o número de UFs eficientes foi superior ao registrado no governo Dilma. Ademais, notou-se que, no Sul, não se registrou nenhuma UF eficiente durante o governo Lula, sendo apenas uma no governo Dilma. Já o Nordeste apresentou maior número de UFs eficientes durante os dois governos, contrastando com o que foi evidenciado pela DEA na modalidade CCR.

As análises apontam resultados divergentes nas duas modalidades da DEA, evidenciando duas diferentes perspectivas acerca do conceito de eficiência. A modalidade CCR considera eficientes aquelas UFs que obedecem à lógica de proporção entre o recurso e o produto, enquanto a modalidade BCC considera eficiente a lógica de economia de recursos.

O teste de Spearman revelou uma correlação positiva entre as modalidades CCR e BCC e entre a modalidade CCR e o PIB, mas não apresentou significância para a modalidade $\mathrm{BCC}$ e o PIB, tanto no governo Lula quanto 


\section{EFICIÊNCIA DA ALOCAÇÃO DE RECURSOS PÚBLICOS NAS UNIDADES DA FEDERAÇÃO NOS GOVERNOS LULA E DILMA}

no governo Dilma.

Ressalte-se que este estudo tem relevância na academia e no âmbito da gestão pública, pois são raros aqueles que verificam a eficiência dos gastos públicos por meio de uma abordagem multidimensional, além da utilização de indicadores de desenvolvimento multidimensional, como o IFDM. Além disso, foram feitas análises comparativas entre os dois citados governos (segundo mandato do presidente Lula e primeiro mandato da presidente Dilma), quanto à eficiência dos gastos públicos e ao desenvolvimento das UFs.

Para futuras pesquisas, sugere-se a investigação da eficiência dos gastos públicos das UFs em outras dimensões. Recomenda-se a utilização de outras variáveis de input (como gastos com segurança pública, assistência social, saneamento) e output (outros indicadores sociais) no modelo DEA, a fim de se ampliarem as dimensões estudadas. Além disso, os modelos e variáveis utilizados neste artigo podem ser adaptados na análise da eficiência de municípios e também de países. Sugere-se, ainda, que estudos futuros possam investigar as dimensões aqui apresentadas (educação, saúde e renda) de maneira separada, considerando a individualidade e a especificidade de cada uma dessas áreas.

\section{REFERÊNCIAS}

Afonso, A., Romero, A., \& Monsalve, E. (2013). Public sector efficiency: Evidence for Latin America [Discussion Paper № IDB-DP-279]. Inter-American Development Bank, Lisboa, Portugal.
Avelino, B. C., Bressan, V. G. F., \& Cunha, J. V. A. (2013). Estudo sobre os fatores contábeis que influenciam o Índice FIRJAN de Desenvolvimento Municipal (IFDM) nas capitais brasileiras. Revista de Educação e Pesquisa em Contabilidade, 7(3), 274-290. doi:10.17524/repec.v7i3.993

Balaguer-Coll, M. T., Prior, D., \& Tortosa-Ausina, E. (2007). On the determinants of local government performance: A two-stage nonparametric approach. European Economic Review, 51(2), 425-451. doi:10.1016/j. euroecorev.2006.01.007

Banker, R. D., Charnes, A., \& Cooper, W. W. (1984). Some models for estimating technical and scale inefficiencies in data envelopment analysis. Management Science, 30(9), 1078-1092. doi:10.1287/mnsc.30.9.1078

Banker, R. D., \& Natarajan, R. (2008). Evaluating contextual variables affecting productivity using data envelopment analysis. Operations Research, 56(1), 48-58. doi:10.1287/ opre. 1070.0460

Beuren, I. M., Moura, G. D., \& Kloeppel, N. R. (2013). Práticas de governança eletrônica e eficiência na utilização das receitas: Uma análise nos estados brasileiros. RAP-Revista Administração Pública, 47(2), 421-441. doi:10.1590/S0034-76122013000200007

Brito, C. C. (2017). Conceito de território rural e políticas públicas e sua evolução histórica nos governos FHC e Lula. Cadernos Gestão Pública e Cidadania, 22(72), 173-192. doi:10.1590/S0034-76122013000200007

Charnes, A., Cooper, W., \& Rodhes, E. (1978). Measuring the efficiency of decision marking 
units. European Journal of Operational Research, 2(6), 429-444. doi:10.1016/03772217(78)90138-8

Chiechelski, P. C. S. (2005). Avaliação de programas sociais: Abordagens quantitativas e suas limitações. Textos \& Contextos, 4(1), 1-12.

Constituição da República Federativa do Brasil de 1988. (1988). Brasília, DF. Recuperado de http://www.planalto.gov.br/ccivil_03/ constituicao/constituicao.htm

Costa, C. C. M., Ferreira, M. A. M., Braga, M. J., \& Abrantes, L. A. (2015). Fatores associados à eficiência na alocação de recursos públicos à luz do modelo de regressão quantílica. RAP-Revista Administração Pública, 49(5), 1319-1347. doi:10.1590/00347612130868

Debnath, R. M., \& Shankar, R. (2014). Does good governance enhance happiness: A cross nation study. Social Indicators Research, 116(1), 235-253. doi:10.1007/s11205013-0275-1

Diniz, J. A., Macedo, M. A. S., \& Corrar, L. J. (2012). Mensuração da eficiência financeira municipal no Brasil e sua relação com os gastos nas funções de governo. Gestão \& Regionalidade, 28(83), 5-20. doi:10.13037/ gr.vol28n83.1347

Faé, R., Goulart, S., \& Abdala, P. R. Z. (2016). Estratégia nacional de desenvolvimento nos governos Lula e Dilma: Transformação neoliberal. Revista Pensamento Contemporâneo em Administração, 10(1), 1-18. doi:10.12712/rpca.v10i1.654
Federação das Indústrias do Estado do Rio de Janeiro. (2015). Índice FIRJAN de desenvolvimento municipal. Publicações Sistemas FIRJAN: Pesquisas e estudos socioeconômicos. Recuperado de http://www.firjan.com.br/ ifdm/downloads/

Federação das Indústrias do Estado do Rio de Janeiro. (2016). Índice FIRJAN de desenvolvimento municipal. Consulta ao índice. Recuperado de http://www.firjan.com.br/ifdm/

Hauner, D., \& Kyobe, A. (2010). Determinants of government efficiency. World Development, 38(11), 1527-1542. doi:10.1016/j.worlddev.2010.04.004

Instituto Brasileiro de Geografia e Estatística. (2016). Estados@. Recuperado de http:// www.ibge.gov.br/estadosat/

Lei Complementar n. 101, de 4 de maio de 2000 (2000). Estabelece normas de finanças públicas voltadas para a responsabilidade na gestão fiscal e dá outras providências. Brasília, DF. Recuperado de http://www.planalto. gov.br/ccivil_03/leis/LCP/Lcp101.htm

Leite, G. A., Filho, \& Fialho, T. M. M. (2015). Relação entre indicadores de gestão pública e de desenvolvimento dos municípios brasileiros. Cadernos Gestão Pública e Cidadania, 20(67), 277-295. doi:10.12660/cgpc. v20n67.52080

Machado, S. P., Júnior, Irffi, G., \& Benegas, M. (2011). Análise da eficiência técnica dos gastos com educação, saúde e assistência social dos municípios cearenses. Planejamento e Políticas Públicas, (36), 87-113.

Meza, A. L., Biondi, L., Neto, Mello, J. C. 
C. B. S., \& Gomes, E. G. (2005). ISYDS - Integrated System for Decision Support (SIAD - Sistema Integrado de Apoio à Decisão): $A$ software package for Data Envelopment Analysis model. Pesquisa Operacional, 25(3), 493-503. doi:10.1590/ S0101-74382005000300011

Morais, L. P. (2015). Desenvolvimento socioeconômico e geração de emprego e renda na América Latina e no Caribe: Potenciais contribuições da economia social e solidária. Revista Direito Mackenzie, 9(1), 131153.

Moura, F. A., \& Sauer, L. (2009, janeiro/abril). Reflexões sobre a metodologia de construção do IDH e suas implicações quantitativas. Desafio: Revista de Economia e Administração, 10(20), 114-128.

Moutinho, J. A. (2016). Transferências voluntárias da União para municípios brasileiros: Mapeamento do cenário nacional. RAP-Revista Administração Pública, 50(1), 151-166. doi:10.1590/0034-7612139003

Mukokoma, M. M. N., \& Dijk, M. P. (2013). New public management reforms and efficiency in urban water service delivery in developing countries: Blessing or fad? Public Works Management \& Policy, 18(1), 23-40. doi:10.1177/1087724X12459043

Peña, C. R. (2008). Um modelo de avaliação da eficiência da administração pública através do método Análise Envoltória de Dados (DEA). RAC-Revista de Administração Contemporânea, 12(1), 83-106. doi:10.1590/ S1415-65552008000100005

Pinto, J. F., \& Santos, L. T. D. (2017). Admi- nistração pública brasileira no século XXI: Caminhamos para alguma reforma? $A d$ ministração Pública e Gestão Social, 9(3), 205-215. doi:10.21118/apgs.v1i3.1325

Rodrigues, M. A. V. (2017). Democracia vs. eficiência: Como alcançar equilíbrio em tempo de crise financeira. RAP-Revista de Administração Pública, 51(1), 88-104. doi:10.1590/0034-7612165400

Sandroni, P. (1989). Dicionário de economia: Nova edição revista e ampliada (8a ed.). São Paulo, SP: Best Seller.

Santos, G. F. Z., \& Schommer, P. C. (2018). Bureaucracy, civil society and ideology in Latin America. Gobernar: The Journal of Latin American Public Policy and Governance, 2(3), 1-17.

Scarpin, J. E., Macedo, F. F. R. R., Starosky, L., Filho, \& Rodrigues, M. M., Júnior. (2012). Análise da eficiência dos recursos públicos direcionados à educação: Estudo nos municípios do estado de Santa Catarina. Revista Gestão Pública: Práticas e Desafios, 3(6), 27-48.

Scarpin, J. E., \& Slomski, V. (2007). Estudo dos fatores condicionantes do Índice de Desenvolvimento Humano nos municípios do estado do Paraná: Instrumento de controladoria para a tomada de decisões na gestão governamental. RAP-Revista Administração Pública, 41(5), 909-933.

Secchi, L. (2009). Modelos organizacionais e reformas da administração pública. RAP-Revista de Administração Pública, 43(2), 347-369. doi:10.1590/S003476122009000200004 
Secretaria do Tesouro Nacional. (2016). Informações contábeis e fiscais do setor público. Recuperado de http://www.tesouro. fazenda.gov.br/web/stn/-/informacoes-contabeis-e-fiscais-do-setor-publico

Siedenberg, R. S. (2003). Indicadores de desenvolvimento socioeconômico: Uma síntese. Desenvolvimento em Questão, 1(1), 4571. doi:10.21527/2237-6453.2003.1.45-71

Silva, M. C., Oliveira, A. F., Martins, J. D. M., \& Silva, J. D. G. (2014). Análise envoltória de dados na avaliação da eficiência das despesas de investimentos dos estados e do Distrito Federal. Revista Universo Contábil, 10(3), 114-133. doi:10.4270/RUC.2014322

Souza, F. J. V., \& Barros, C. C. (2013). Eficiência na alocação de recursos públicos destinados a assistência hospitalar nos estados brasileiros. Revista de Gestão, Finanças e Contabilidade, 3(1), 71-89. doi:10.29386/ rgfc.v3i1.84

Souza, P. C., Scatena, J. H. G., \& Kehrig, R. T. (2017). Eficiência hospitalar no SUS: Análise de 10 hospitais do mix público-privado do Estado de Mato Grosso. Cadernos Gestão Pública e Cidadania, 22(72), 335-354. doi:10.12660/cgpc.v22n72.66242

Trompieri-Neto, N., Lopes, D. A. F., Barbosa, M. P., \& Holanda, M. C. (2009). Determinantes da eficiência dos gastos públicos municipais em educação e saúde: $O$ caso do Ceará. In E. B. S. Carvalho, M. C. Holanda, \& M. P. Barbosa (Orgs.), Economia do Ceará em debate (pp. 57-72). Fortaleza, CE: IPECE. 